\title{
Spectrum of mutations in the familial Mediterranean fever gene $(M E F V)$ in Turkish patients of the Central Anatolia region: a comparison of two mutation detection system
}

\author{
A.G. Zamani, A. Acar and M.S. Yildirim \\ Department of Medical Genetics, Meram Medical Faculty, \\ Necmettin Erbakan University, Meram, Konya, Turkey \\ Corresponding author: A.G. Zamani \\ E-mail:agzamani@yahoo.com
}

Genet. Mol. Res. 12 (4): 5152-5159 (2013)

Received March 13, 2013

Accepted August 30, 2013

Published October 29, 2013

DOI http://dx.doi.org/10.4238/2013.October.29.9

ABSTRACT. The purpose of this study was to determine the spectrum
of the most common mutations in the familial Mediterranean fever
gene (MEFV) in Turkish patients from the Central Anatolia region, by
using two different methods for detecting FMF-associated mutations
with different screening panels, and compare our results with other
diagnostic molecular genetics centers. A total of 1579 patients were
analyzed. Genomic DNA from 304 patients was tested for 6 common
mutations located in exon 2 (E148Q), and exon 10 (M680I, M694V,
M694I, V726A, R761H) by real-time PCR while 1275 patients were
tested for 17 mutations located in exon 2 (E148Q), and exon10 [M680I
(G/C), M680I (G/A), I692del, M694V, M694I, K695R, V726A, S675N,
G678E, M680L, T681I, M694L, K695M, R717S, I720M, V722M] by
pyrosequencing. The most frequent mutation was M694V, followed
by M680I, E148Q, and V726A. Ten mutations in the panel were not
detected in any patients. Finally, we compared our results with those
of other centers in Turkey to contribute to the identified spectrum of 
Turkish $M E F V$ mutations and we discuss which $M E F V$ mutations are informative for evaluating an FMF patient.

Key words: $M E F V$ gene; FMF; Mutation; Molecular diagnosis

\section{INTRODUCTION}

Familial Mediterranean fever (FMF, OMIM 249100) is an autosomal recessive genetic disease that is characterized by recurrent episodes of autoinflammatory fever and abdominal pain, synovitis and pleuritis. The most severe manifestation of the disease is renal amyloidosis. FMF mainly affects people of eastern Mediterranean origin, especially non-Ashkenazi Jews, Armenians, Turks, and Arabs. The disease is unusual in other populations, but it has been described in Greeks, Italians, Cubans, and Belgians (Wekell et al., 2013). The gene responsible for the condition $M E F V$ has been mapped to chromosome 16p13.3 (Medlej-Hashim et al., 2010). It consists of 10 exons, which encode the 781-residue protein pyrin/marenostrin. This protein is expressed in granulocytes and thought to be a negative regulator of inflammation. More than 60 or the $186 M E F V$ mutations that have been detected in affected individuals have been shown to influence the disease (French FMF Consortium, 1997). A recent study has shown that, although MEFV mutations are major genetic determinants of FMF, there may be a number of other genes involved in FMF (Soriano and Manna, 2012).

The overall prevalence of the disease in the Turkish population has been estimated as 1 in 1073 (Ozen et al., 1998). The carrier frequency is $1 / 5$ in the Turkish population (Yilmaz et al., 2009). Turkey, with a population of more than 70 million, has a large proportion of all the FMF cases in the world. For this reason, many centers in Turkey routinely screen for FMF mutations using various methods. Screening panels that detect 12 mutations are used in most of the centers. Five key mutations (M694V, V726A, M680I and M694I (in exon 10) and E148Q (in exon 2) screened for by almost all of the FMF research centers, together with other less common mutations (Jarjour, 2010).

This particular disease is one of the major monogenic diseases that have been tested for, since the founding of the Diagnostic Molecular Genetics Center at the Meram Medical Faculty. In this study, we discuss the results of cases analyzed using two different methods over the last two years. We evaluate the molecular test results from 1579 patients (304 of them analyzed with a 6-mutation panel and 1275 with a 17 -mutation panel) that were referred to our department for $M E F V$ analysis.

The purpose of this study was to evaluate two different mutation panels and compare our data with those of the other centers in Turkey that screen for the disease with panels of the most extensive series of mutations. We aim to contribute to the Turkish MEFV mutations spectrum data and discuss the necessity of including rare mutations in the detection panels.

\section{MATERIAL AND METHODS}

Mutation analyses of the MEFV gene were performed in a total of 1579 patients referred to our genetic polyclinic with the clinical diagnosis of FMF from different clinics over the last two years. DNA was extracted from $2 \mathrm{~mL}$ of peripheral blood that had been collected into EDTA-anticoagulated tubes, using the QIAamp DNA Blood Isolation kit (Qiagen GmbH) 
following the manufacturer protocol. The DNA concentration was determined using a NanoDrop spectrophotometer (Thermo Scientific, USA). Mutation analysis of the MEFV gene was performed by two different methods: real-time PCR and pyrosequencing.

Genomic DNA from patients was tested for the 6 common mutations located in exon 2 (E148Q), and exon 10 (M680I, M694V, M694I, V726A,R761H) by using real-time PCR. PCR amplification was performed in $20-\mu \mathrm{L}$ volumes in glass capillaries, in the presence of $2 \mathrm{mM}$ $\mathrm{MgCl}_{2}$ and $1 \mu \mathrm{L}$ each of primers and mutation probe. The probes hybridized head-to-tail, bringing the two fluorescent dyes into proximity. Fluorescence was monitored while the temperature was slowly increased; the fluorescence decreased when one of the probes melted off and the fluorescent dyes were no longer in proximity. The typical melting pattern with the wild-type DNA sequence is a single melting peak at a characteristic, reproducible temperature. In cases with homozygous mutations, the anticipated mismatch leads to hybrid instability and, as a consequence, earlier melting. The result is a single melting peak at a characteristic, lower temperature, whereas samplesfrom patients with heterozygous mutations show two melting peaks.

For pyrosequencing analysis, all samples underwent testing for 17 mutations located in exon 2 (E148Q), and exon 10 [M680I (G/C), M680I (G/A), I692del, M694V, M694I, K695R, V726A, S675N, G678E, M680L, T681I, M694L, K695M, R717S, I720M,V722M] in accordance with the manufacturer protocol. The pyrosequencing kits use a biotinylated reverse primer, allowing the isolation of single stranded templates (of the reverse strand) from the PCR products. The following materials and reagents were used: binding buffer (Qiagen), streptavidin Sepharose high-performance beads (GE Biosciences, Uppsala, Sweden), and sterile water. The product was eluted using at PyroMark Vacuum Prep Work-Station (Qiagen). Pyro-sequencing reactions were carried out with a nested sequencing primer in a PyroMark MD (Qiagen) and PyroGold Reagents (Qiagen) in accordance with the manufacturer protocol. The results were analyzed using the software Pyro Q-CpG (Qiagen).

\section{RESULTS}

A total of 1579 patients were referred to our genetic polyclinic with the clinical diagnosis of FMF from different clinics and were analyzed with either the 6- or 17-mutation panel. The 6-mutation panel was used in 304 cases, while 17-mutations panel was used in 1275 cases. No mutations were found in $150(50.7 \%)$ of the patients from the group screened for 6 mutations and in $658(51.60 \%)$ of the patients from the group screened for 17 mutations.

In the 6-mutations panel group, $81(54.0 \%)$ patients were heterozygotes, $37(24.6 \%)$ were compound heterozygotes and $31(20.6 \%)$ were homozygotes for the mutation. The most common heterozygous genotypes were E148Q/- (21.3\%), M694V/- (20.6\%), M680I/- (6.7\%), and V726A/- (4.0\%). The most common compound heterozygous genotypes were M694V/ M680I (6.7\%), and M694V/E148Q (5.3\%). The distribution of patients homozygous for a mutation in this group were as follows: 18 (12\%) M694V/M694V, 12 (8\%) M680I/M680I, and $1(0.6 \%) \mathrm{E} 148 \mathrm{Q} / \mathrm{E} 148 \mathrm{Q}$. A complex genotype was detected in only one patient .

Mutations were identified in 617 patients of the 17-mutation screened group. The distribution of genotype frequencies was as follows: 431 patients $(69.8 \%)$ were heterozygotes, $122(18.1 \%)$ patients were compound heterozygotes and $74(12.1 \%)$ patients were homozygous for the mutation. The most common heterozygous genotypes were M694V/-(26.8\%), E148Q/- (19.6\%), V726A/- (9.5\%), and M680I/- (8.2\%) (The sum of G/Cand G/Amutations). 
The M694V/V726A genotype was the most common type of compound heterozygote (5.3\%). The distribution of cases of homozygotes in this group was as follows: $(10.1 \%) \mathrm{M} 694 \mathrm{~V} /$ M694V, (2.4\%) M680I/M680I, and 1 (0.6\%) E148Q/E148Q.

Mutant alleles across both groups, no mutation was found in the second group but not in the first group, except for K695R (5.4\% of cases). Of all the patients observed with mutations $512(65.0 \%)$ had at least one mutant allele, $149(19,3 \%)$ were compound heterozygotes and $105(13.8 \%)$ were homozygotes for the mutation. The most frequently observed heterozygotes were M694V/- (23.9\%) and E148Q/- (20.4\%). The M680I/- mutations(M680I, M680I G/C, and M680I G/A, together) were the third frequent, followed by genotype V726A/(6.9\%). Genotypes M694V/V726A (4.4\%), M694V/E148Q (3.8\%), and M694V/M680I were the most common compound heterozygous genotypes. Most of the patients with homozygous mutations had a the M694V/M694V (11\%)genotype.

The incidence of M680I (20.64\%) and E148Q (20.18\%) mutations was very similar. K695R, M694I and R761H mutations were seen rare (2.06, 1.38, 0.92\% respectively), and mutations M680L, T681I, 1692del, M694L, K695M, R717S, I720M and V722M were found in none of the patients tested (Figure 1).

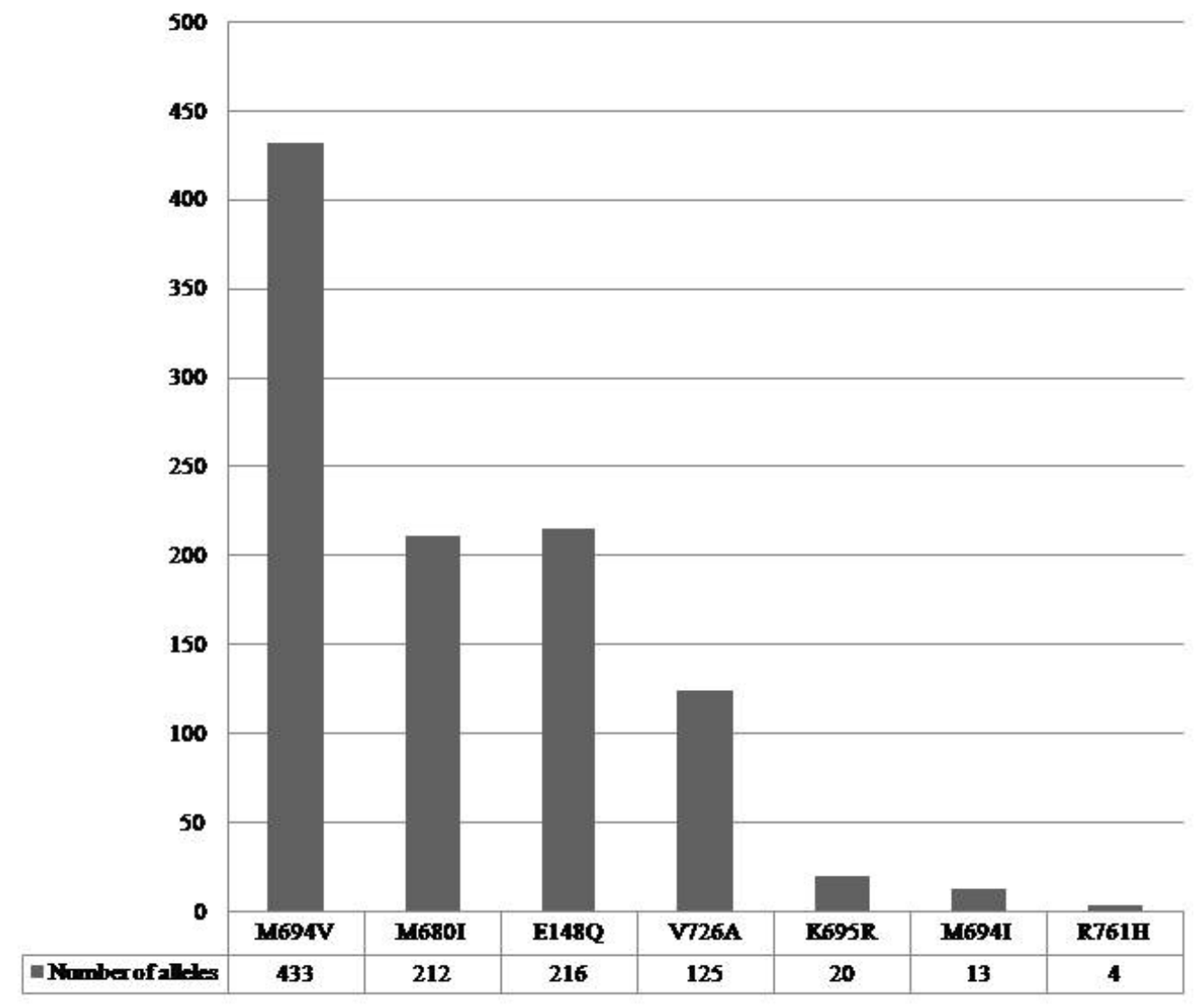

Figure 1. Allelic distribution of patients. 


\section{DISCUSSION}

FMF is the most prevalent periodic fever syndrome, affecting more than 100,000 patients worldwide (Fonnesu et al., 2009). The minimal and most current criteria for diagnosis of FMF are the Tel Hashomer clinical criteria. These criteria for the disease stipulate fever plus one other major symptome and one minor symptome, or fever plus two minor symptoms (Livneh et al., 1997). The major symptoms are fever, abdominal pain, chest pain, skin eruption, and joint pain. The minor signs are an increased erythrocyte sedimentation rate, leukocytosis, and an elevated serum concentration of fibrinogen (Shohat and Halpern, 2011). FMF notoriously difficult to diagnose until mutations in the $M E F V$ gene were identified, which are differently associated with the severity of the disease phenotype. Over 80 mutations in $M E F V$ have been detected in FMF patients and registered in the auto-inflammatory mutation database Infevers http://fmf.igh.cnrs.fr/infevers/ (Medlej-Hashim et al., 2010).

Therefore, MEFV mutations are under investigation in several clinical centers in the eastern Mediterranean region where the incidence of FMF is high. However, $M E F V$ mutations can be detected in a defined group of patients clinically diagnosed as having FMF. Thus, $M E F V$ mutations were detected only in $(51.5 \%)$ of 1579 cases examined in our study and no mutations were detected in the remaining (48.5\%). We suggest that this high rate at which previously unknown mutations have been identified may be attributed to an increased usage of molecular tests in the differential diagnosis of symptoms similar to those seen in FMF. In the Central Anatolia Region, most physicians are aware of the high frequency of FMF in the local population. Therefore, FMF is one of the most likely initial diagnoses in most patients having unexplained fever and abdominal pain. However, some of these patients may have mutations that are not in our analysis spectrum or they may have mutations in other FMF-causing genes. There are many reports where a high proportion of patients present FMF symptoms without having a known mutation (Fonnesu et al., 2009). Sabbagh et al. also reported a high rate (51.5\%) of FMF cases without mutations among Lebanese patients that were referred for FMF molecular analysis (Sabbagh et al., 2008). The distribution of FMF-related mutations varies depending on ethnicity and geography. Therefore, we compared the incidence of mutations detected in 1579 cases of this study with the results of the four largest studies in Turkey (Figure 2).

In our study, in the middle of Anatolia, M694V was the most common mutation. This situation seemed to be harmonious with the findings of the other four studies. The allelic frequency of the M694V mutation was $42.3 \%$ in our study. It ranged between $43.12 \%$ and $47.60 \%$ in the other studies. The homozygous genotype frequency of this mutation is compatible with the findings of other studies (11.0-16.6\%).

Heterozygous M694V genotypes are the most frequently detected group of genotypes in our study. The frequency of consanguineous marriages is twenty-five percent in our region and it is likely that this has contributed to the high frequency of homozygosity at M694V. In our study, the next most common mutations were, M680I and E148Q. Mutations of G/C and G/A could not be distinguished at M680I in the first panel that we used. Thus, G/C and G/A mutations at M680I were screened by the second panel and evaluated under the name of M680I. The total frequency of M680I was $20.7 \%$ in this study, and very similar to the frequency of mutations at E148Q (21.1\%). While mutations at M680I are the third and fourth most frequently reported in studies from the Aegean region (Berdeli at al., 2011), they were the second and third most frequent in studies from Central Anatolia (Dundar et al., 2011). 


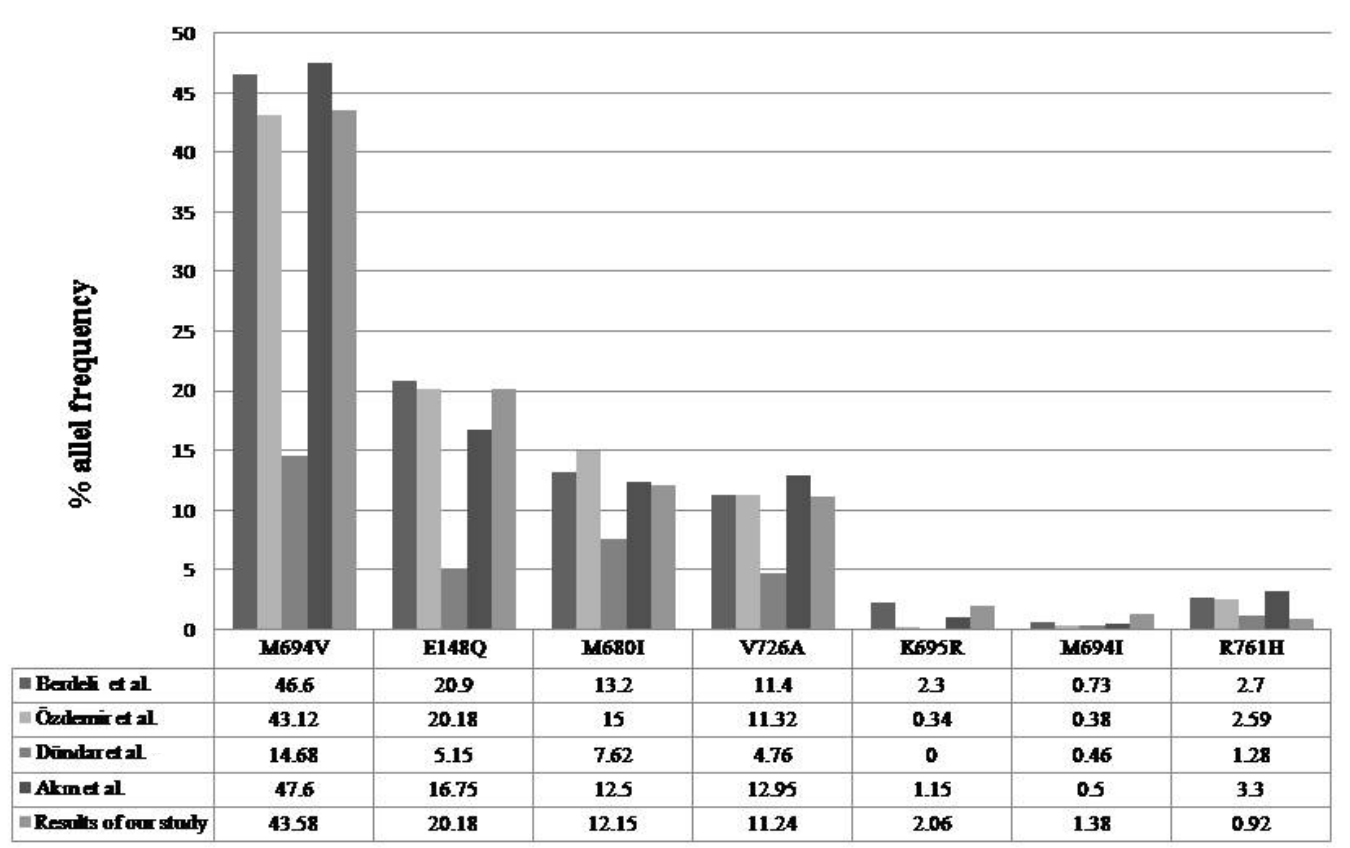

Figure 2. Comparison of our results with other regional study.

From these findings, it is evident that, similarities in the type of mutation identified in FMF is associated with geographical proximity. Mutation V726A was the fourth most frequently encountered in our study, and mutation K695R was the fifth most frequently encountered in our study. Conversely, P369S was the fifth most frequent mutation in two of the four other studies, and R761H was fifth in the other two. Mutation K695R ranked in $7^{\text {th }}-10^{\text {th }}$ most frequent in the other studies. However, we did not include P369S and A744S in our panels of screened mutations. A744S was ranked in the other studies in $6^{\text {th }}-10^{\text {th }}$ place. The $\mathrm{R} 761 \mathrm{H}$ mutation was the seventh most frequent in our study. Mutation M694I, which ranks sixth in our study, was ranked in $7^{\text {th }}-10^{\text {th }}$ most frequent in the other studies. These comparisons lead us to the conclusion that mutations K695R, M694I, and R761H with allele frequencies (2.06, 1.38, 0.92\%, respectively) are rare mutations in our study region.

Mutations S675N, G678E, M680L, T681I, 1692del, M694L, K695M, R717S, I720M, and V722M were not detected in our study. Therefore, these alleles dare likely to be rare in the Central Anatolia Region. The screened panels of the four comparable studies did not include these mutations except for $1692 \mathrm{del}$ so, we have no further evidence to support this hypothesis. The 1692del mutation was detected in only one of the cases in the comparable studies. This supports the view that this allele is a very rare.

FMF is a common disease among people orginating from the Mediterranean region and there is evidence that the mutations in the $M E F V$ gene have different frequency distributions in different ethnic groups. We compared the frequencies of the mutant alleles in our study from the Central Anatolia Region with the studies of countries in the Mediterranean belt. The M694V mutation is the most common mutation in Turkey, Lebanon, Syria, Jordan, Azerbaijan 
and Armenia. In contrast, M694I is the most common mutation in Egypt and Morocco, but is rarely found in Turkey. The most common mutation reported from Tunisia was M680I which was the third place in our study. The fourth most frequent mutation in our study, V726A was the second in Lebanon, Syria, Jordan, Azerbaijan and Armenia. This mutation was the fifth most frequent in studies of Morocco and Tunisia (Figure 3).

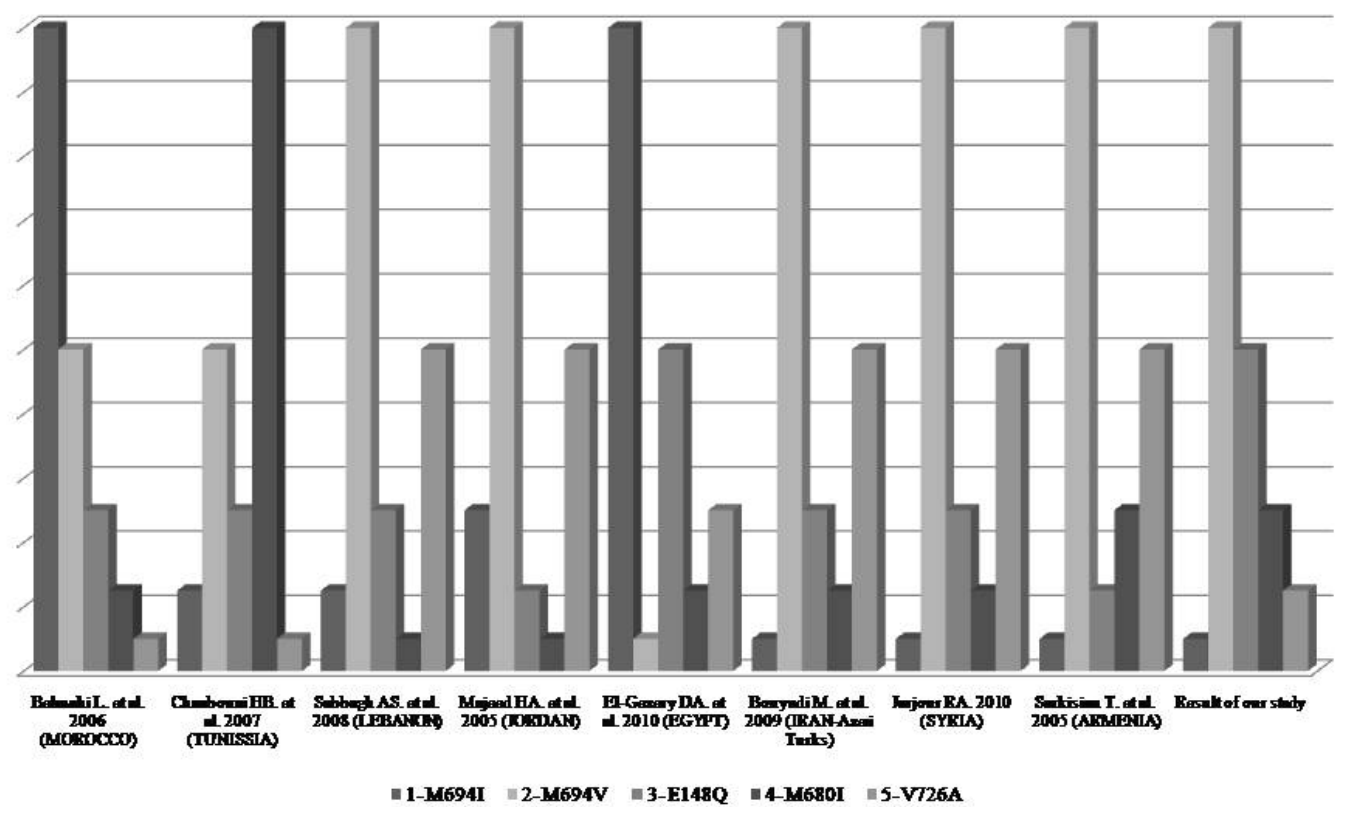

Figure 3. Comparison of our mutation results with other countries studies.

In conclusion, clinicians in the countries of the Mediterranean belt where the FMF is an important health problem should apply the diagnostic criteria of FMF more frequently in the clinical diagnosis of FMF. Furthermore, centers that analyze FMF mutations must take into account the geographical and ethnic background of patients to identify which mutations should be screened. These recommendations will result in a more efficient use of the time and resources avaliable for the diagnosis of FMF. Furthermore, the research for previously undetected mutations associated with FMF must be continued in $M E F V$ gene and in other genes.

\section{REFERENCES}

Akin H, Onay H, Turker E, Cogulu O, et al. (2010). MEFV mutations in patients with Familial Mediterranean Fever from the Aegean region of Turkey. Mol. Biol. Rep. 37: 93-98.

Berdeli A, Mir S, Nalbantoglu S, Kutukculer N, et al. (2011). Comprehensive analysis of a large-scale screen for MEFV gene mutations: do they truly provide a "heterozygote advantage" in Turkey? Genet. Test. Mol. Biomarkers 15: 475-482.

Dundar M, Emirogullari EF, Kiraz A, Taheri S, et al. (2011). Common Familial Mediterranean Fever gene mutations in a Turkish cohort. Mol. Biol. Rep. 38: 5065-5069.

Fonnesu C, Cerquaglia C, Giovinale M, Curigliano V, et al. (2009). Familial Mediterranean fever: a review for clinical management. Joint Bone Spine 76: 227-233. 
French FMF Consortium (1997). A candidate gene for familial Mediterranean fever. Nat. Genet. 17: 25-31.

Jarjour RA (2010). Familial Mediterranean fever in Syrian patients: MEFV gene mutations and genotype-phenotype correlation. Mol. Biol. Rep. 37: 1-5.

Livneh A, Langevitz P, Zemer D, Zaks N, et al. (1997). Criteria for the diagnosis of familial Mediterranean fever. Arthritis Rheum. 40: 1879-1885.

Medlej-Hashim M, Nehme N, Chouery E, Jalkh N, et al. (2010). 1 Novel MEFV transcripts in familial Mediterranean fever patients and controls. BMC Med. Genet. 11: 87.

Ozen S, Karaaslan Y, Ozdemir O, Saatci U, et al. (1998). Prevalence of juvenile chronic arthritis and familial Mediterranean fever in Turkey: a field study. J. Rheumatol. 25: 2445-2449.

Sabbagh AS, Ghasham M, Abdel KR, Greije L, et al. (2008). MEFV gene mutations spectrum among Lebanese patients referred for familial Mediterranean fever work-up: experience of a major tertiary care center. Mol. Biol. Rep. 35: 447-451.

Shohat M and Halpern GJ (2011). Familial Mediterranean fever - a review. Genet. Med. 13: 487-498.

Soriano A and Manna R (2012). Familial Mediterranean fever: new phenotypes. Autoimmun. Rev. 12: 31-37.

Wekell P, Friman V, Balci-Peynircioglu B, Yilmaz E, et al. (2013). Familial Mediterranean fever - an increasingly important childhood disease in Sweden. Acta Paediatr. 102: 193-198.

Yilmaz R, Ozer S, Ozyurt H, Erkorkmaz U, et al. (2009). Familial Mediterranean fever gene mutations in the inner northern region of Turkey and genotype-phenotype correlation in children. J. Paediatr. Child Health 45: 641-645. 